November 2008

SLAC-PUB-13454

\title{
Dynamic versus Static Structure Functions and Novel Diffractive Effects in QCD
}

\author{
Stanley J. Brodsky \\ SLAC National Accelerator Laboratory Stanford University, Stanford, CA 94309, USA \\ Institute for Particle Physics and Phenomenology, Durham, UK
}

\begin{abstract}
Initial- and final-state rescattering, neglected in the parton model, have a profound effect in QCD hard-scattering reactions, predicting single-spin asymmetries, diffractive deep inelastic scattering, diffractive hard hadronic reactions, the breakdown of the Lam Tung relation in DrellYan reactions, and nuclear shadowing and non-universal antishadowing-leading-twist physics not incorporated in the light-front wavefunctions of the target computed in isolation. I also discuss the use of diffraction to materialize the Fock states of a hadronic projectile and test QCD color transparency, and anomalous heavy quark effects. The presence of direct higher-twist processes where a proton is produced in the hard subprocess can explain the large proton-to-pion ratio seen in high centrality heavy ion collisions. I emphasize the importance of distinguishing between static observables such as the probability distributions computed from the square of the light-front wavefunctions versus dynamical observables which include the effects of rescattering.
\end{abstract}

Keywords: Diffraction, AdS/CFT, QCD, Holography, Light-Front Wavefunctions, Multiple Scattering, Heavy-Ion Collisions

PACS: $12.38 . \mathrm{Aw}, 24.85 .+\mathrm{p}, 25.75 . \mathrm{Bh}, 12.40 . \mathrm{Nn}, 11.15 . \mathrm{Tk}, 11.80 . \mathrm{La}$

\section{NOVEL FEATURES OF DIFFRACTIVE DEEP INELASTIC SCATTERING}

A remarkable feature of deep inelastic lepton-proton scattering at HERA is that approximately $10 \%$ events are diffractive [1, 2]: the target proton remains intact, and there is a large rapidity gap between the proton and the other hadrons in the final state. This observation presents a paradox: if one chooses the conventional parton model frame, the virtual photon interacts with a quark constituent with light-cone momentum fraction $x=k^{+} / p^{+}=x_{b j}$. Furthermore, the gauge link associated with the struck quark (the Wilson line) becomes unity in light-cone gauge $A^{+}=0$. Thus the struck "current" quark apparently experiences no final-state interactions. Since the light-front wavefunctions $\psi_{n}\left(x_{i}, \mathbf{k}_{\perp i}\right)$ of a stable hadron are real, it appears impossible to generate the required imaginary phase associated with pomeron exchange, let alone large rapidity gaps. This paradox was resolved by Hoyer, Marchal, Peigne, Sannino and myself [3]. Consider the case where the virtual photon interacts with a strange quark-the $s \bar{s}$ pair is assumed to be produced in the target by gluon splitting. In the case of Feynman gauge, the struck $s$ quark continues to interact in the final state via gluon exchange as described by the Wilson line. The final-state interactions occur at a light-cone time $\Delta \tau \simeq 1 / v$ shortly after the virtual photon interacts with the struck quark. When one integrates over the nearly-on-shell intermediate state, the amplitude acquires an imaginary part. Thus the rescattering of the quark produces a separated color-singlet $s \bar{s}$ and an imaginary phase.

Invited talk presented at the Diffraction 2008: International Workshop On Diffraction In High Energy Physics, 9/9/2008 to 9/14/2008, La Londe-les-Maures, France

Work supported in part by US Department of Energy contract DE-AC02-76SF00515 
In the case of the light-cone gauge $A^{+}=\eta \cdot A=0$, one must also consider the final-state interactions (rescattering) of the (unstruck) $\bar{s}$ quark. The gluon propagator in light-cone gauge is inversely proportional to $k^{+}$. The momentum of the exchanged gluon $k^{+}$is of $\mathscr{O}(1 / v)$; thus rescattering contributes at leading twist even in light-cone gauge. The net result is gauge invariant and identical to a color dipole model calculation. The calculation of the rescattering effects on DIS in Feynman and light-cone gauge through three loops is given in detail for an Abelian model in ref. [3]. The result shows that the rescattering corrections reduce the magnitude of the DIS cross section in analogy to nuclear shadowing.

A new understanding of the role of rescattering in deep inelastic scattering has thus emerged. The multiple scattering of the struck parton via instantaneous interactions in the target generates dominantly imaginary diffractive amplitudes, giving rise to an effective "hard pomeron" exchange. The presence of a rapidity gap between the target and diffractive system requires that the target remnant emerges in a color-singlet state; this is made possible in any gauge by the soft rescattering. The resulting diffractive contributions leave the target intact and do not resolve its quark structure; thus there are contributions to the DIS structure functions which cannot be interpreted as parton probabilities [3]; the leading-twist contribution to DIS from rescattering of a quark in the target is a coherent effect which is not included in the light-front wavefunctions computed in isolation.

Another novel QCD phenomenon involving nuclei is the antishadowing of the nuclear structure functions which is observed in deep inelastic lepton scattering and other hard processes. Empirically, one finds $R_{A}\left(x, Q^{2}\right) \equiv\left(F_{2 A}\left(x, Q^{2}\right) /(A / 2) F_{d}\left(x, Q^{2}\right)\right)>1$ in the domain $0.1<x<0.2$; i.e., the measured nuclear structure function (referenced to the deuteron) is larger than than the scattering on a set of $A$ independent nucleons. Ivan Schmidt, Jian-Jun Yang, and I [4] have extended the analysis of nuclear shadowing to the shadowing and antishadowing of all of the electroweak structure functions. We note that there are also leading-twist diffractive contributions $\gamma^{*} N_{1} \rightarrow(q \bar{q}) N_{1}$ arising from Reggeon exchanges in the $t$-channel [5]. For example, isospin-non-singlet $C=$ + Reggeons contribute to the difference of proton and neutron structure functions, giving the characteristic Kuti-Weisskopf $F_{2 p}-F_{2 n} \sim x^{1-\alpha_{R}(0)} \sim x^{0.5}$ behavior at small $x$. The $x$ dependence of the structure functions reflects the Regge behavior $v^{\alpha_{R}(0)}$ of the virtual Compton amplitude at fixed $Q^{2}$ and $t=0$. The phase of the diffractive amplitude is determined by analyticity and crossing to be proportional to $-1+i$ for $\alpha_{R}=0.5$, which together with the phase from the Glauber cut, leads to constructive interference of the diffractive and nondiffractive multi-step nuclear amplitudes. The nuclear structure function is predicted to be enhanced precisely in the domain $0.1<$ $x<0.2$ where antishadowing is empirically observed. The strength of the Reggeon amplitudes is fixed by the fits to the nucleon structure functions, so there is little model dependence. Quarks of different flavors will couple to different Reggeons; this leads to the remarkable prediction that nuclear antishadowing is not universal; it depends on the quantum numbers of the struck quark. This picture implies substantially different antishadowing for charged and neutral current reactions, thus affecting the extraction of the weak-mixing angle $\theta_{W}$. We find that part of the anomalous NuTeV result [6] for $\theta_{W}$ could be due to the non-universality of nuclear antishadowing for charged and neutral 


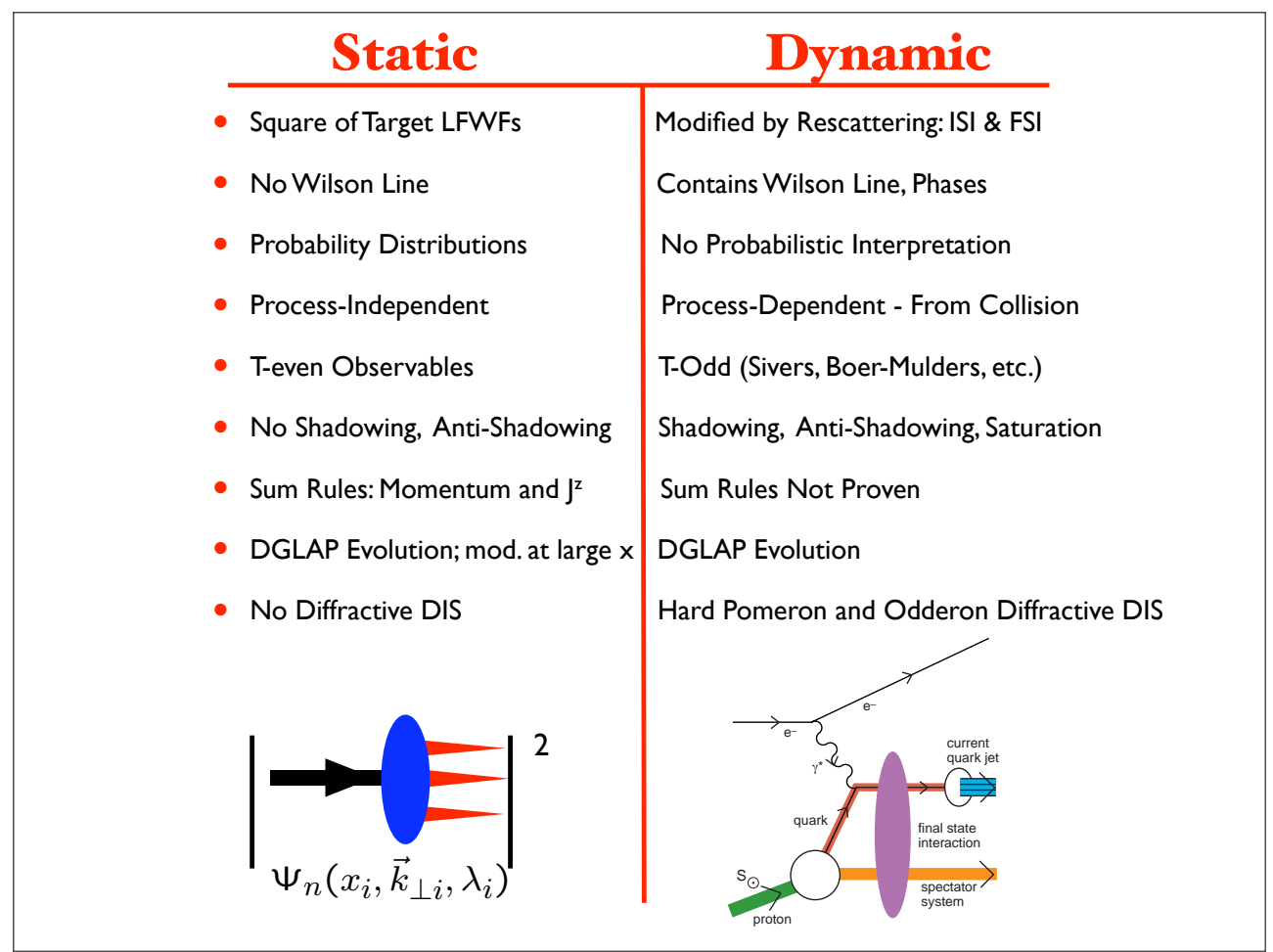

FIGURE 1. Static versus dynamic structure functions

currents. In fact, Schienbein et al. [7] have recently given a comprehensive analysis of charged current deep inelastic neutrino-iron scattering, finding significant differences with the nuclear corrections for electron-iron scattering.

It is thus important to distinguish "static" structure functions which are computed directly from the LFWFs of the target from the "dynamic" empirical structure functions which take into account rescattering of the struck quark. Since they derive from the LF eigenfunctions of the target hadron, the static structure functions have a probabilistic interpretation. Since the wavefunction of a stable eigenstate is real, the static structure functions do not describe DDIS nor the single-spin asymmetries discussed below since such phenomena involves the complex phase structure of the $\gamma^{*} p$ amplitude. One can augment the light-front wavefunctions with a gauge link corresponding to an external field created by the virtual photon $q \bar{q}$ pair current [8, 9], but such a gauge link is process dependent [10], so the resulting augmented wavefunctions are not universal. [3, 8, 11]. We emphasize that the shadowing of nuclear structure functions is due to the destructive interference between multi-nucleon amplitudes involving diffractive DIS and on-shell intermediate states with a complex phase. The physics of rescattering and shadowing is thus not included in the nuclear light-front wavefunctions, and a probabilistic interpretation of the nuclear DIS cross section is precluded. The distinction between static structure functions; i.e., the probability distributions computed from the square of the light-front wavefunctions, versus the nonuniversal dynamic structure functions measured in deep inelastic scattering is summarized in fig. 1 . 


\section{SINGLE-SPIN ASYMMETRIES AND OTHER LEADING-TWIST RESCATTERING EFFECTS}

Among the most interesting polarization effects are single-spin azimuthal asymmetries in semi-inclusive deep inelastic scattering, representing the correlation of the spin of the proton target and the virtual photon to hadron production plane: $\vec{S}_{p} \cdot \vec{q} \times \vec{p}_{H}$. Such asymmetries are time-reversal odd, but they can arise in QCD through phase differences in different spin amplitudes. In fact, final-state interactions from gluon exchange between the outgoing quarks and the target spectator system lead to single-spin asymmetries (SSAs) in semi-inclusive deep inelastic lepton-proton scattering which are not powerlaw suppressed at large photon virtuality $Q^{2}$ at fixed $x_{b j}$ [12]. In contrast to the SSAs arising from transversity and the Collins fragmentation function, the fragmentation of the quark into hadrons is not necessary; one predicts a correlation with the production plane of the quark jet itself. Physically, the final-state interaction phase arises as the infrared-finite difference of QCD Coulomb phases for hadron wavefunctions with differing orbital angular momentum. The same proton matrix element which determines the spin-orbit correlation $\vec{S} \cdot \vec{L}$ also produces the anomalous magnetic moment of the proton, the Pauli form factor, and the generalized parton distribution $E$ which is measured in deeply virtual Compton scattering. Thus the contribution of each quark current to the SSA is proportional to the contribution $\kappa_{q / p}$ of that quark to the proton target's anomalous magnetic moment $\kappa_{p}=\sum_{q} e_{q} \kappa_{q / p}$ [12, 13]. The HERMES collaboration has recently measured the SSA in pion electroproduction using transverse target polarization [14]. A related analysis also predicts that the initial-state interactions from gluon exchange between the incoming quark and the target spectator system lead to leadingtwist single-spin asymmetries in the Drell-Yan process $H_{1} H_{2}^{\uparrow} \rightarrow \ell^{+} \ell^{-} X$ [10, 15]. The SSA in the Drell-Yan process is the same as that obtained in SIDIS, with the appropriate identification of variables, but with the opposite sign. There is no such single spin asymmetries in charged-current reactions since the $W$ only couples to left-handed quarks [16]. If both the quark and antiquark in the initial state of the Drell-Yan subprocess $q \bar{q} \rightarrow \mu^{+} \mu^{-}$interact with the spectators of the other incident hadron, one finds a breakdown of the Lam-Tung relation, which was formerly believed to be a general prediction of leading-twist QCD. These double initial-state interactions also lead to a $\cos 2 \phi$ planar correlation in unpolarized Drell-Yan reactions [17].

As noted by Collins and Qiu [18], the traditional factorization formalism of perturbative QCD for high transverse momentum hadron production fails in detail even at the LHC because of initial- and final-state rescattering. The signal for factorization breakdown is a $\cos 2 \phi$ planar correlation in dijet production.

\section{NOVEL HEAVY QUARK PHENOMENA}

The probability for Fock states of a light hadron such as the proton to have an extra heavy quark pair decreases as $1 / m_{Q}^{2}$ in non-Abelian gauge theory [19, 20]. The relevant matrix element is the cube of the QCD field strength $G_{\mu v}^{3}$. This is in contrast to abelian gauge theory where the relevant operator is $F_{\mu \nu}^{4}$ and the probability of intrinsic heavy 
leptons in QED bound state is suppressed as $1 / m_{\ell}^{4}$. The intrinsic Fock state probability is maximized at minimal off-shellness. It is useful to define the transverse mass $m_{\perp i}=$ $\sqrt{k_{\perp i}^{2}+m_{i}^{2}}$. The maximum probability then occurs at $x_{i}=m_{\perp}^{i} / \sum_{j=1}^{n} m_{\perp}^{j}$; i.e., when the constituents have minimal invariant mass and equal rapidity. Thus the heaviest constituents have the highest momentum fractions and the highest $x_{i}$. Intrinsic charm thus predicts that the charm structure function has support at large $x_{b j}$ in excess of DGLAP extrapolations [21]; this is in agreement with the EMC measurements [22]. Intrinsic charm can also explain the $J / \psi \rightarrow \rho \pi$ puzzle [23]. It also affects the extraction of suppressed CKM matrix elements in $B$ decays [24]. The dissociation of the intrinsic charm $\mid u u d c \bar{c}>$ Fock state of the proton on a nucleus can produce a leading heavy quarkonium state at high $x_{F}=x_{c}+x_{\bar{c}}$ in $p A \rightarrow J / \psi X A^{\prime}$ since the $c$ and $\bar{c}$ can readily coalesce into the charmonium state. Since the constituents of a given intrinsic heavyquark Fock state tend to have the same rapidity, coalescence of multiple partons from the projectile Fock state into charmed hadrons and mesons is also favored. For example, one can produce a leading $\Lambda_{c}$ at high $x_{F}$ and low $p_{T}$ from the coalescence of the $u d c$ constituents of the projectile $\mid u u d c \bar{c}>$ Fock state.

In the case of a nuclear target, the charmonium state will be produced at small transverse momentum and high $x_{F}$ with a characteristic $A^{2 / 3}$ nuclear dependence since the color-octet color-octet $\mid(u u d)_{8 C}(c \bar{c})_{8 C}>$ Fock state interacts on the front surface of the nuclear target [25]. This forward contribution is in addition to the $A^{1}$ contribution derived from the usual perturbative QCD fusion contribution at small $x_{F}$. Because of these two components, the cross section violates perturbative QCD factorization for hard inclusive reactions [26]. This is consistent with the observed two-component cross section for charmonium production observed by the NA3 collaboration at CERN [27] and more recent experiments [28]. The diffractive dissociation of the intrinsic charm Fock state leads to leading charm hadron production and fast charmonium production in agreement with measurements [29]. The hadroproduction cross sections for double-charm $\Xi_{c c}^{+}$baryons at SELEX [30] and the production of $J / \psi$ pairs at NA3 are be consistent with the diffractive dissociation and coalescence of double IC Fock states [31]. These observations provide compelling evidence for the diffractive dissociation of complex off-shell Fock states of the projectile and contradict the traditional view that sea quarks and gluons are always produced perturbatively via DGLAP evolution. It is also conceivable that the observations [32] of $\Lambda_{b}$ at high $x_{F}$ at the ISR in high energy $p p$ collisions could be due to the diffractive dissociation and coalescence of the "intrinsic bottom" $\mid u u d b \bar{b}>$ Fock states of the proton. As emphasized by Lai, Tung, and Pumplin [33], there are strong indications that the structure functions used to model charm and bottom quarks in the proton at large $x_{b j}$ have been strongly underestimated, since they ignore intrinsic heavy quark fluctuations of hadron wavefunctions.

Goldhaber, Kopeliovich, Schmidt, Soffer, and I [25, 34] have proposed a novel mechanism for exclusive diffractive Higgs production $p p \rightarrow p H p$ and nondiffractive Higgs production in which the Higgs boson carries a significant fraction of the projectile proton momentum. The production mechanism is based on the subprocess $(Q \bar{Q}) g \rightarrow H$ where the $Q \bar{Q}$ in the $\mid u u d Q \bar{Q}>$ intrinsic heavy quark Fock state has up to $80 \%$ of the projectile protons momentum. This mechanism provides a clear experimental signal for Higgs production at the LHC due to the small background in this kinematic region. 


\section{DIFFRACTION DISSOCIATION AS A TOOL TO RESOLVE HADRON SUBSTRUCTURE AND TEST COLOR TRANSPARENCY}

Diffractive multi-jet production in heavy nuclei provides a novel way to resolve the shape of light-front Fock state wavefunctions and test color transparency [35]. For example, consider the reaction [36, 37]. $\pi A \rightarrow \mathrm{Jet}_{1}+\mathrm{Jet}_{2}+A^{\prime}$ at high energy where the nucleus $A^{\prime}$ is left intact in its ground state. The transverse momenta of the jets balance so that $\vec{k}_{\perp i}+\vec{k}_{\perp 2}=\vec{q}_{\perp}<R_{A}^{-1}$. Because of color transparency, the valence wavefunction of the pion with small impact separation will penetrate the nucleus with minimal interactions, diffracting into jet pairs [36]. The $x_{1}=x, x_{2}=1-x$ dependence of the dijet distributions will thus reflect the shape of the pion valence light-cone wavefunction in $x$; similarly, the $\vec{k}_{\perp 1}-\vec{k}_{\perp 2}$ relative transverse momenta of the jets gives key information on the second transverse momentum derivative of the underlying shape of the valence pion wavefunction [37, 38]. The diffractive nuclear amplitude extrapolated to $t=0$ should be linear in nuclear number $A$ if color transparency is correct. The integrated diffractive rate will then scale as $A^{2} / R_{A}^{2} \sim A^{4 / 3}$. This is in fact what has been observed by the E791 collaboration at FermiLab for $500 \mathrm{GeV}$ incident pions on nuclear targets [39].

Light-Front Holography is one of the most remarkable features of AdS/CFT [40, 41, 42]. It allows one to project the functional dependence of the wavefunction $\Phi(z)$ computed in the AdS fifth dimension to the hadronic frame-independent light-front wavefunction $\psi\left(x_{i}, \mathbf{b}_{\perp i}\right)$ in $3+1$ physical space-time. The variable $z$ maps to $\zeta\left(x_{i}, \mathbf{b}_{\perp i}\right)$. To prove this, we have shown that there exists a correspondence between the matrix elements of the energy-momentum tensor of the fundamental hadronic constituents in QCD with the transition amplitudes describing the interaction of string modes in antide Sitter space with an external graviton field which propagates in the AdS interior. The agreement of the results for both electromagnetic and gravitational hadronic transition amplitudes provides an important consistency test and verification of holographic mapping from AdS to physical observables defined on the light-front [43]. In fact $\zeta$ is the only variable to appear in the light-front Schrödinger equations predicted from AdS/QCD [40]. These equations for both meson and baryons give a good representation of the observed hadronic spectrum, especially in the case of the soft wall model. The resulting LFWFs also have excellent phenomenological features, including predictions for the electromagnetic form factors and decay constants. We have also shown that the LF Hamiltonian formulation of quantum field theory provides a natural formalism to compute hadronization at the amplitude level [44]. It is interesting to note that the form of the nonperturbative pion distribution amplitude $\phi_{\pi}(x)$ obtained from integrating the prediction from AdS/QCD $q \bar{q}$ valence LFWF $\psi\left(x, \mathbf{k}_{\perp}\right)$ over $\mathbf{k}_{\perp}$, has a quite different $x$-behavior [45] than the asymptotic distribution amplitude predicted from PQCD evolution [46]. The AdS/QCD prediction $\phi_{\pi}(x)=\sqrt{3} f_{\pi} \sqrt{x(1-x)}$ has a broader distribution than expected from solving the ERBL evolution equation in perturbative QCD. This observation appears to be consistent with the results of the Fermilab diffractive dijet experiment [47] in the low $p_{T}$ regime, the moments obtained from lattice QCD [48] and pion form factor data [49]. 


\section{COLOR TRANSPARENCY AND THE RHIC BARYON ANOMALY}

It is conventional to assume that leading-twist subprocesses dominate measurements of high $p_{T}$ hadron production at RHIC energies. Indeed the data for direct photon fragmentation $p p \rightarrow \gamma X$ is quite consistent with $n_{e f f}(p p \rightarrow \gamma X)=5$, as expected from the $g q \rightarrow \gamma q$ leading-twist subprocess. This also is likely true for pion production, at least for small $x_{T}$. However, the measured fixed $x_{T}$ scaling for proton production at RHIC is anomalous: PHENIX reports $n_{e f f}(p p \rightarrow p X) \simeq 8$. A review of this data is given by Tannenbaum [50]. One can understand the anomalous scaling if a higher-twist subprocess [51] where the proton is made directly within the hard reaction, such as $u u \rightarrow p \bar{d}$ and $(u u d) u \rightarrow p u$, dominates the reaction $p p \rightarrow p X$ at RHIC energies. Such processes are rigorous QCD contributions. The dominance of direct subprocesses is possible since the fragmentation of gluon or quark jets to baryons requires that the 2 to 2 subprocess occurs at much higher transverse momentum than the $p_{T}$ of observed proton because of the fast-falling $(1-z)^{3}$ quark-to-proton fragmentation function. Such "direct" reactions can readily explain the fast-falling power-law falloff observed at fixed $x_{T}$ and fixed $-\theta_{c m}$ observed at the ISR, FermiLab and RHIC. Furthermore, the protons produced directly within the hard subprocess emerge as small-size color-transparent colored states which are not absorbed in the nuclear target. In contrast, pions produced from jet fragmentation have the normal cross section. This provides a plausible explanation of the RHIC data, [52] which shows a dramatic rise of the $p \rightarrow \pi$ ratio at high $p_{T}$ when one compares peripheral with central (full overlap) heavy ion collisions. The directly produced protons are not absorbed, but the pions are diminished in the nuclear medium.

\section{ACKNOWLEDGMENTS}

Presented at the Diffraction 2008: International Workshop On Diffraction In High Energy Physics 9-14 Sep 2008, La Londe-les-Maures, France. I thank Paul Hoyer, Ivan Schmidt, Boris Kopeliovich, Dae Sung Hwang, Fred Goldhaber, Jacques Soffer, Anne Sickles, Ramona Vogt, Daniel Boer and Guy de Teramond for our collaborations. I also am grateful to Jacques Soffer, and Roberto Fiore for their hospitality at Diffraction 2008.

SLAC-PUB-13454 This research was supported by the Department of Energy contract DE-AC02-76SF00515.

\section{REFERENCES}

1. C. Adloff et al. [H1 Collaboration], Z. Phys. C 76, 613 (1997) [arXiv:hep-ex/9708016].

2. J. Breitweg et al. [ZEUS Collaboration], Eur. Phys. J. C 6, 43 (1999) [arXiv:hep-ex/9807010].

3. S. J. Brodsky, P. Hoyer, N. Marchal, S. Peigne and F. Sannino, Phys. Rev. D 65, 114025 (2002) [arXiv:hep-ph/0104291].

4. S. J. Brodsky, I. Schmidt and J. J. Yang, Phys. Rev. D 70, 116003 (2004) [arXiv:hep-ph/0409279].

5. S. J. Brodsky and H. J. Lu, Phys. Rev. Lett. 64, 1342 (1990).

6. G. P. Zeller et al. [NuTeV Collaboration], Phys. Rev. Lett. 88, 091802 (2002) [Erratum-ibid. 90, 239902 (2003)] [arXiv:hep-ex/0110059].

7. I. Schienbein, J. Y. Yu, C. Keppel, J. G. Morfin, F. I. Olness and J. F. Owens, arXiv:0806.0723 [hep-ph]. 
8. A. V. Belitsky, X. Ji and F. Yuan, Nucl. Phys. B 656, 165 (2003) [arXiv:hep-ph/0208038].

9. J. C. Collins and A. Metz, Phys. Rev. Lett. 93, 252001 (2004) [arXiv:hep-ph/0408249].

10. J. C. Collins, Phys. Lett. B 536, 43 (2002) [arXiv:hep-ph/0204004].

11. J. C. Collins, Acta Phys. Polon. B 34, 3103 (2003) [arXiv:hep-ph/0304122].

12. S. J. Brodsky, D. S. Hwang and I. Schmidt, Phys. Lett. B 530, 99 (2002) [arXiv:hep-ph/0201296].

13. M. Burkardt, Nucl. Phys. Proc. Suppl. 141, 86 (2005) [arXiv:hep-ph/0408009].

14. A. Airapetian et al. [HERMES Collaboration], Phys. Rev. Lett. 94, 012002 (2005) [arXiv:hepex/0408013].

15. S. J. Brodsky, D. S. Hwang and I. Schmidt, Nucl. Phys. B 642, 344 (2002) [arXiv:hep-ph/0206259].

16. S. J. Brodsky, D. S. Hwang and I. Schmidt, Phys. Lett. B 553, 223 (2003) [arXiv:hep-ph/0211212].

17. D. Boer, S. J. Brodsky and D. S. Hwang, Phys. Rev. D 67, 054003 (2003) [arXiv:hep-ph/0211110].

18. J. Collins and J. W. Qiu, Phys. Rev. D 75, 114014 (2007) [arXiv:0705.2141 [hep-ph]].

19. M. Franz, M. V. Polyakov and K. Goeke, Phys. Rev. D 62, 074024 (2000) [arXiv:hep-ph/0002240].

20. S. J. Brodsky, J. C. Collins, S. D. Ellis, J. F. Gunion and A. H. Mueller, Proc. of 1984 Summer Study on the SSC, Snowmass, CO, Published in Snowmass Summer Study 1984:0227 (QCD184:S7:1984)

21. S. J. Brodsky, P. Hoyer, C. Peterson and N. Sakai, Phys. Lett. B 93, 451 (1980).

22. B. W. Harris, J. Smith and R. Vogt, Nucl. Phys. B 461, 181 (1996) [arXiv:hep-ph/9508403].

23. S. J. Brodsky and M. Karliner, Phys. Rev. Lett. 78, 4682 (1997) [arXiv:hep-ph/9704379].

24. S. J. Brodsky and S. Gardner, Phys. Rev. D 65, 054016 (2002) [arXiv:hep-ph/0108121].

25. S. J. Brodsky, B. Kopeliovich, I. Schmidt and J. Soffer, Phys. Rev. D 73, 113005 (2006) [arXiv:hep$\mathrm{ph} / 0603238]$.

26. P. Hoyer, M. Vanttinen and U. Sukhatme, Phys. Lett. B 246, 217 (1990).

27. J. Badier et al. [NA3 Collaboration], Phys. Lett. B 104, 335 (1981).

28. M. J. Leitch et al. [FNAL E866/NuSea collaboration], Phys. Rev. Lett. 84, 3256 (2000) [arXiv:nuclex/9909007].

29. J. C. Anjos, J. Magnin and G. Herrera, Phys. Lett. B 523, 29 (2001) [arXiv:hep-ph/0109185].

30. A. Ocherashvili et al. [SELEX Collaboration], Phys. Lett. B 628, 18 (2005) [arXiv:hep-ex/0406033].

31. R. Vogt and S. J. Brodsky, Phys. Lett. B 349, 569 (1995) [arXiv:hep-ph/9503206].

32. G. Bari et al., Nuovo Cim. A 104, 1787 (1991).

33. J. Pumplin, H. L. Lai and W. K. Tung, Phys. Rev. D 75, 054029 (2007) [arXiv:hep-ph/0701220].

34. S. J. Brodsky, A. S. Goldhaber, B. Z. Kopeliovich and I. Schmidt, Nucl. Phys. B 807, 334 (2009) [arXiv:0707.4658 [hep-ph]].

35. S. J. Brodsky and A. H. Mueller, Phys. Lett. B 206, 685 (1988).

36. G. Bertsch, S. J. Brodsky, A. S. Goldhaber and J. F. Gunion, Phys. Rev. Lett. 47, 297 (1981).

37. L. Frankfurt, G. A. Miller and M. Strikman, Found. Phys. 30, 533 (2000) [arXiv:hep-ph/9907214].

38. N. N. Nikolaev, W. Schafer and G. Schwiete, Phys. Rev. D 63, 014020 (2001) [arXiv:hep$\mathrm{ph} / 0009038]$

39. E. M. Aitala et al. [E791 Collaboration], Phys. Rev. Lett. 86, 4773 (2001) [arXiv:hep-ex/0010044].

40. G. F. de Teramond and S. J. Brodsky, arXiv:0809.4899 [hep-ph].

41. S. J. Brodsky and G. F. de Teramond, arXiv:0804.3562 [hep-ph].

42. S. J. Brodsky and G. F. de Teramond, arXiv:0810.1876 [hep-ph].

43. S. J. Brodsky and G. F. de Teramond, Phys. Rev. D 78, 025032 (2008) [arXiv:0804.0452 [hep-ph]].

44. S. J. Brodsky, G. de Teramond and R. Shrock, AIP Conf. Proc. 1056, 3 (2008) [arXiv:0807.2484 [hep-ph]].

45. S. J. Brodsky and G. F. de Teramond, Phys. Rev. Lett. 96, 201601 (2006) [arXiv:hep-ph/0602252].

46. G. P. Lepage and S. J. Brodsky, Phys. Lett. B 87, 359 (1979).

47. E. M. Aitala et al. [E791 Collaboration], Phys. Rev. Lett. 86, 4768 (2001) [arXiv:hep-ex/0010043].

48. S. J. Brodsky and G. F. de Teramond, arXiv:0802.0514 [hep-ph].

49. H. M. Choi and C. R. Ji, Phys. Rev. D 74, 093010 (2006) [arXiv:hep-ph/0608148].

50. M. J. Tannenbaum, PoS C FRNC2006, 001 (2006) [arXiv:nucl-ex/0611008].

51. S. J. Brodsky and A. Sickles, Phys. Lett. B 668, 111 (2008) [arXiv:0804.4608 [hep-ph]].

52. S. S. Adler et al. [PHENIX Collaboration], Phys. Rev. Lett. 91, 172301 (2003) [arXiv:nuclex/0305036]. 